\title{
Characterization of FeSe thin films prepared on GaAs substrate by selenization technique
}

\author{
Y. Takemura, H. Suto, N. Honda, and K. Kakuno \\ Division of Electrical and Computer Engineering, Yokohama National University, 156 Tokiwadai, \\ Hodogaya, Yokohama 240, Japan \\ K. Saito \\ Department of Electronics and Information Sciences, Teikyo University of Science and Technology, \\ 2525 Yatsuzawa, Uenohara, Yamanashi 409-01, Japan
}

FeSe thin films were prepared on $\operatorname{GaAs}(100)$ substrate by the selenization of Fe films using molecular-beam epitaxy. FeSe compound thin films were obtained at a substrate temperature above $380{ }^{\circ} \mathrm{C}$. From the depth profiles of $\mathrm{Fe}$ and Se in the selenized film measured by Auger electron spectroscopy, it was confirmed that an FeSe layer with a constant ratio of $\mathrm{Fe} / \mathrm{Se}$ was formed. The measured composition ratio of $\mathrm{Fe} / \mathrm{Se}$ in the film was $1 / 3$. It was different from the composition in $\mathrm{Fe}_{3} \mathrm{Se}_{4}$ or $\mathrm{Fe}_{7} \mathrm{Se}_{8}$, which is a stable bulk FeSe compound. From the measured $M-H$ curve, it was found that the obtained FeSe film consisted of two phases with different magnetic properties.

(C) 1997 American Institute of Physics. [S0021-8979(97)31508-4]

\section{INTRODUCTION}

Magnetic semiconductors including Mn-related materials and semiconductor/magnetic metal hybrid structures have recently attracted much attention as new magnetic materials and their applications for electronic devices. Among them, $\mathrm{Fe} / \mathrm{ZnSe}$ and $\mathrm{FeSe} / \mathrm{ZnSe}$ systems are interesting because their thin films can be grown on a GaAs substrate. ${ }^{1-3}$ In this article fabrication and characterization of FeSe thin films are reported. FeSe thin films are expected to be utilized as an interlayer for semiconductor/ferromagnetic metal heterostructures, or as a new magnetic material. Characterizations of bulk crystals of FeSe have been reported with $\mathrm{Fe}_{3} \mathrm{Se}_{4}$ and $\mathrm{Fe}_{7} \mathrm{Se}_{8}$ structures, ${ }^{4,5}$ but FeSe thin films have not been studied much. The lattice structure of bulk $\mathrm{Fe}_{3} \mathrm{Se}_{4}$ and $\mathrm{Fe}_{7} \mathrm{Se}_{8}$ is $\mathrm{NiAs}$ type with ordered $\mathrm{Fe}$ vacancies. The origin of the ferrimagnetism of these materials is attributed to this ordered arrangement of $\mathrm{Fe}$ vacancies similar to the case of $\mathrm{Fe}_{7} \mathrm{Se}_{8}$ pyrrhotite. Curie temperatures of $\mathrm{Fe}_{3} \mathrm{Se}_{4}$ and $\mathrm{Fe}_{7} \mathrm{Se}_{8}$ are 314 and $455 \mathrm{~K}$, respectively. Because of their lattice structures, they have a strong magneto-crystalline anisotropy along the $c$ axis.

\section{EXPERIMENTS}

FeSe thin films were prepared on GaAs substrate by the selenization technique. A semiinsulating nondoped $\operatorname{GaAs}(100)$ substrate was used. Before the deposition of the Fe film, the substrate was chemically etched in a solution of $3 \mathrm{H}_{2} \mathrm{SO}_{4}+\mathrm{H}_{2} \mathrm{O}_{2}+\mathrm{H}_{2} \mathrm{O}$ at $60^{\circ} \mathrm{C}$ for $1.5 \mathrm{~min}$, followed by successive rinses of acetone and methanol. The $\mathrm{Fe}$ thin film deposited on GaAs substrates by vacuum evaporation or sputtering was selenized in a molecular-beam-epitaxy (MBE) chamber as shown in Fig. 1. Conventional MBE equipment with solid sources was used. A typical thickness of the Fe films was $235 \mathrm{~nm}$. The selenization was performed by supplying a Se beam from a $K$ cell at substrate temperatures ranging from 260 to $530{ }^{\circ} \mathrm{C}$ for $1-4 \mathrm{~h}$. The Se beam pressure was varied from $2 \times 10^{-8}$ to $8 \times 10^{-8}$ Torr.
The thickness and depth profiles of $\mathrm{Fe}$ and $\mathrm{Se}$ of the selenized films were evaluated in order to confirm the formation of FeSe. Auger electron spectroscopy (AES) was used for the measurement of the depth profiles. Electron dispersive $\mathrm{x}$-ray spectroscopy (EDX) was used to determine the $\mathrm{Fe} / \mathrm{Se}$ ratio in the films. As for magnetic properties of the films, $M-H$ curves were measured with a vibrating sample magnetometer (VSM) at room temperature.

\section{RESULTS AND DISCUSSION}

The thickness of the selenized films was measured. Because the vapor pressure of $\mathrm{Se}$ is as high as 0.1 Torr at $250{ }^{\circ} \mathrm{C}$, the Se atom itself is not deposited on the Fe films by supplying a Se beam to the Fe films on GaAs substrate at substrate temperatures above $250{ }^{\circ} \mathrm{C}$. The increase of the $\mathrm{Fe}$ film thickness by the selenization process is only caused by the formation of an FeSe compound. Figure 2 shows the total thickness of the films selenized for $1 \mathrm{~h}$ with Se beam pressure of $2 \times 10^{-8}$ Torr at the various substrate temperatures. The total thickness is defined as described in Fig. 1. As the thickness of the Fe film before the selenization is $235 \mathrm{~nm}$, selenization of the $\mathrm{Fe}$ does not proceed below the substrate temperature of $320^{\circ} \mathrm{C}$. It indicates that the substrate temperature is not high enough for the diffusion of Se atoms and reaction of $\mathrm{Fe}$ and $\mathrm{Se}$. Above $380{ }^{\circ} \mathrm{C}$ the film thickness in-

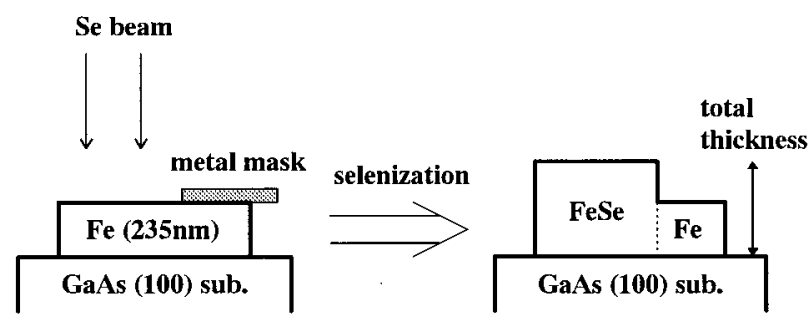

FIG. 1. Schematic diagrams of the selenization process of Fe film on GaAs(100) substrate to prepare FeSe thin film in the molecular-beamepitaxy equipment. 


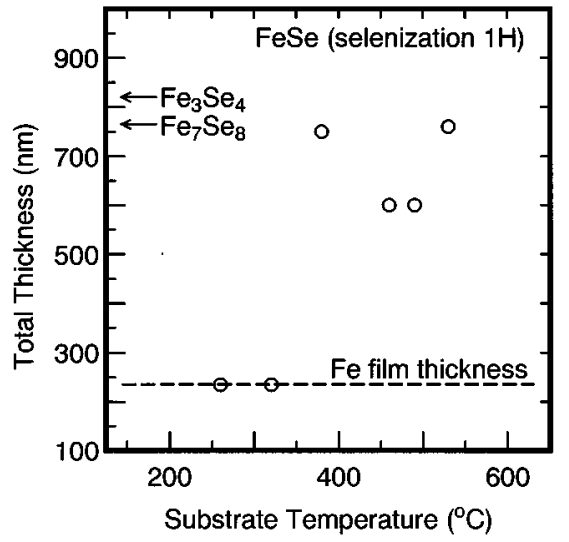

FIG. 2. Total thickness of the films selenized for $1 \mathrm{~h}$ with Se beam pressure of $2 \times 10^{-8}$ Torr at substrate temperatures of $260-530^{\circ} \mathrm{C}$. The thickness of the Fe film before the selenization is $235 \mathrm{~nm}$. Arrows indicate the calculated values assuming the formation of NiAs-type $\mathrm{Fe}_{3} \mathrm{Se}_{4}$ or $\mathrm{Fe}_{7} \mathrm{Se}_{8}$.

creases, which can be understood by the formation of FeSe compound. The complex dependence of the total thickness on the substrate temperature is explained by the selenization rate and the reevaporation of $\mathrm{Se}$ atoms. The selenization rate is considered to increase with an increase in substrate temperature. It is because the selenization process consists of the diffusion of Se atoms and the chemical reaction of $\mathrm{Fe}$ and Se. On the other hand, with increasing substrate temperature the number of the reevaporated $\mathrm{Se}$ atoms increases, which results in a decrease of the selenization rate.

As for bulk FeSe compounds, $\mathrm{Fe}_{3} \mathrm{Se}_{4}$ and $\mathrm{Fe}_{7} \mathrm{Se}_{8}$ with a NiAs-type structure are stable. In Fig. 2 the calculated values of the total thickness are also indicated. In the calculation it is assumed that the whole Fe layer is selenized and that NiAs-type $\mathrm{Fe}_{3} \mathrm{Se}_{4}$ or $\mathrm{Fe}_{7} \mathrm{Se}_{8}$ are formed. The lattice parameters of $a=0.354 \mathrm{~nm}, c=0.558 \mathrm{~nm}$ for $\mathrm{Fe}_{3} \mathrm{Se}_{4}$, and $a=0.362$ $\mathrm{nm}, c=0.589 \mathrm{~nm}$ for $\mathrm{Fe}_{7} \mathrm{Se}_{8}$ are used, respectively. ${ }^{4,5}$ Thicknesses of the obtained films are not so different from the calculated values, but the structure of the films can not be determined from the figure.

Figure 3 shows the depth profiles of $\mathrm{Fe}$ and $\mathrm{Se}$ of the selenized film measured by AES. The sample was selenized at a substrate temperature of $380{ }^{\circ} \mathrm{C}$ with a Se beam pressure of $4 \times 10^{-8}$ Torr for $4 \mathrm{~h}$. The total thickness of the film was $250 \mathrm{~nm}$. As shown in the figure, the profile pattern consists of two regions: region (a) and region (b). They correspond to the regions from the surface to the depth of $100 \mathrm{~nm}$ and from the depth of $100 \mathrm{~nm}$ to the substrate, respectively. In region (a) signals from $\mathrm{Fe}$ and $\mathrm{Se}$ are detected and it indicates that the whole Fe layer is selenized. The intensities of signals of $\mathrm{Fe}$ and Se are constant, which indicates that the layer consists of FeSe compound with a constant composition ratio of $\mathrm{Fe} / \mathrm{Se}$. If the layer consists of FeSe alloylike structure, the intensity of the AES signal of Se should decrease with increasing depth from the surface. The measured value of the composition rate of $\mathrm{Fe} / \mathrm{Se}$ in this sample from the EDX measurement was $1 / 3$. The result indicates that $\mathrm{FeSe}$ layer in region (a) is a Se-rich structure comparable to $\mathrm{Fe}_{3} \mathrm{Se}_{4}$ or $\mathrm{Fe}_{7} \mathrm{Se}_{8}$.

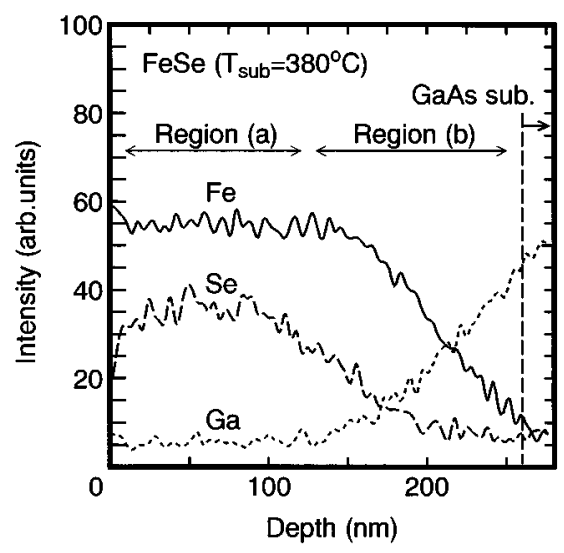

FIG. 3. Depth profiles of Fe and Se measured from the selenized film of 250 $\mathrm{nm}$ thickness by Auger electron spectroscopy. Selenization was performed for $4 \mathrm{~h}$ with Se beam pressure of $4 \times 10^{-8}$ Torr at the substrate temperature of $380^{\circ} \mathrm{C}$.

In region (b) the signal intensities of $\mathrm{Fe}$ and Se decrease toward the interface of the film/GaAs substrate. Since a signal from Se is observed near the interface, it is found that the whole Fe layer is selenized. The decreases of the signal intensities of $\mathrm{Fe}$ and $\mathrm{Se}$ are caused by the diffusion of $\mathrm{Ga}$ from GaAs substrate. In order to avoid the diffusion of Ga atoms, the duration of the selenization process should be reduced.

The measured $M-H$ curve of an FeSe film of $600 \mathrm{~nm}$ thickness is shown in Fig. 4. The sample was selenized at a substrate temperature of $460{ }^{\circ} \mathrm{C}$ with a Se beam pressure of $2 \times 10^{-8}$ Torr for $1 \mathrm{~h}$. The external magnetic field $H$ was applied along the GaAs [011] axis (in plain of the film). The saturation magnetization $M_{S}$ and remnant magnetization of the film are 120 and $41 \mathrm{emu} / \mathrm{cm}^{3}$, respectively. The obtained $M_{S}$ is larger than the reported value of $M_{S}$ in bulk $\mathrm{Fe}_{7} \mathrm{Se}_{8}(65$ $\mathrm{emu} / \mathrm{cm}^{3}$ at $\left.290 \mathrm{~K}\right){ }^{4}$ The $M-H$ curve indicates that the sample consists of two phases with their coercive forces of about 300 and 2200 Oe. As for bulk $\mathrm{Fe}_{3} \mathrm{Se}_{4}$ and $\mathrm{Fe}_{7} \mathrm{Se}_{8}$ of NiAs- type structures, it has been reported that the $c$-axis

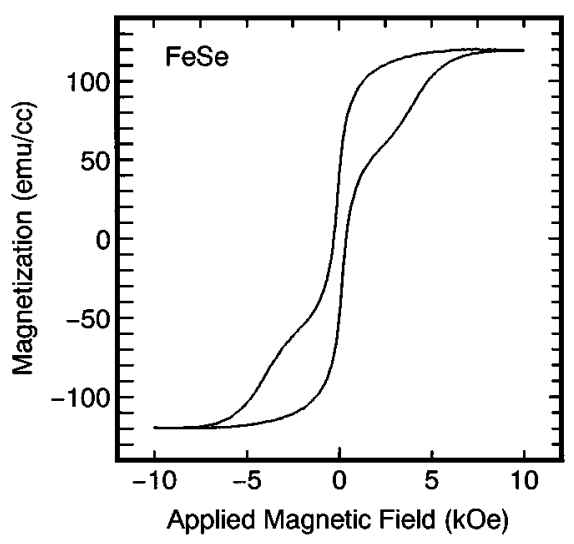

FIG. 4. $M-H$ curve measured on the selenized film of $600 \mathrm{~nm}$ thickness with a vibrating sample magnetometer. Selenization was performed for $1 \mathrm{~h}$ with Se beam pressure of $2 \times 10^{-8}$ Torr at a substrate temperature of $460{ }^{\circ} \mathrm{C}$. The external magnetic field $H$ was applied along GaAs [011] axis (in plain of the film). 
direction is magnetically hard and that the in-plain direction, perpendicular to the $c$ axis, is magnetically soft. The double phase structure observed in the $M-H$ characteristic of the sample is presumably attributed to the mixed structures, for example, regions (a) and (b), as obtained in the AES profile, or to the mixed crystal orientation of the film. Although the reflection high-energy electron diffraction (RHEED) pattern of the sample indicates that the film is polycrystalline, the crystal orientation has not been determined.

\section{CONCLUSION}

FeSe thin films were prepared on GaAs(100) substrate by the selenization technique. After the selenization process the thickness of the film increased, which corresponded to the formation of FeSe compound. By AES depth analysis it was confirmed that the Fe layer was selenized and that a FeSe layer with a constant ratio of $\mathrm{Fe} / \mathrm{Se}$ was formed. The composition ratio of $\mathrm{Fe} / \mathrm{Se}$ in $\mathrm{FeSe}$ film was measured to be $1 / 3$ by the EDX measurement. Since bulk FeSe, $\mathrm{Fe}_{3} \mathrm{Se}_{4}$, and $\mathrm{Fe}_{7} \mathrm{Se}_{8}$ have been reported to be stable compounds, there is a possibility that a different crystal structure of FeSe thin film was obtained from the selenization process. From the measured $M-H$ curve the obtained FeSe film consisted of two phases with different coercive forces. These results indicate that the obtained FeSe thin films have different structural and magnetic properties from bulk FeSe compounds.

\section{ACKNOWLEDGMENTS}

The authors would like to acknowledge Professor M. Konagai at the Tokyo Institute of Technology for the EDX measurement and Professor M. Naoe and Dr. N. Matsushita at the Tokyo Institute of Technology for the VSM measurement.

${ }^{1}$ B. T. Jonker, J. J. Krebs, G. A. Prinz, and S. B. Qadri, J. Cryst. Growth 81, 524 (1987).

${ }^{2}$ K. Park, L. Salamanca-Riba, and B. T. Jonker, 40th MMM Conference 1995, paper CQ-03.

${ }^{3}$ T. Abe, T. Kondo, Y. Shigeno, H. Munekata, J. Abe, H. Ikekame, and K. Sato, in Spring Meeting Jpn. Soc. Appl. Phys., 1996, paper 29a-W-7.

${ }^{4}$ T. Kamimura, K. Kamigaki, T. Hirose, and K. Sato, J. Phys. Soc. Jpn. 22, 1235 (1967)

${ }^{5}$ A. F. Andresen, Acta Chem. Scand. 22, 827 (1968). 